\title{
Synthesis, Spectral Characterization and Kinetic Study of Cu(II) Complex with $N$-Hexahydrocyclopenta[c]pyrrol-2(1H)-yl carbamoyl-4-methylbenzenesulfonamide
}

\author{
BAL KRISHAN $^{* 1}$ and S. A. IQBAL ${ }^{2}$ \\ ${ }^{1}$ Department of Chemistry, Saifia Science College, Barkatullah University, Bhopal -462001, India \\ ${ }^{2}$ Department of Chemistry, Crescent College of Technology, RGPV, Bhopal- 462 00, India \\ bkrishan.krg@gmail.com
}

Received 2 May 2013 / Accepted 7 June 2013

\begin{abstract}
Metal complex of anti-diabetic drug, gliclazide was synthesized and characterized using elemental analyses, IR, NMR and thermal analyses technique. The results of conductometric titration using monovariation method indicate that complex is $\mathrm{L}_{2} \mathrm{M}(2: 1)$ type which was confirmed by Job's method of continuous variation. The complex having the general formula i.e. $\left(\mathrm{C}_{15} \mathrm{H}_{20} \mathrm{~N}_{3} \mathrm{O}_{3} \mathrm{~S}\right){ }_{2} \mathrm{Cu}$. The geometry of complex is assigned square planner supported by IR and ${ }^{1} \mathrm{H}$ NMR and propose structure (Scheme 2) for complex. The Freeman-Carroll and Sharp-Wentworth methods have been used to calculate activation energy and thermal stability. Thermodynamic parameters such as free energy change $(\Delta G)$, entropy change $(\Delta S)$ are also determined on the basis of TG curves and by using data of the Freeman-Carroll method and relative thermal stability of the complex are discussed.
\end{abstract}

Keywords: Gliclazide, Oral anti-diabetic drugs, Gliclazide, Kinetic parameter

\section{Introduction}

Gliclazide (Figure 1) is a sulphonylurea derivatives can exist in keto and enolic form when dissolved in an organic solvent and react with various metal ions to form intensely coloured metal complexes that provide the basis for their use as a sensitive reagent. The thermal degradation study of complexes has become a subject of recent interest. It is important property of complexes, which decides the thermal stability and processability of the complexes. The study of thermal behavior of complexes at different temperature provides important information about its practical applicability. Iqbal et $a 1^{l}$., synthesized the metal complexes of gliclazide characterized by FT-IR, elemental analysis and TGA-DTG parameters. The thermal analysis (TGA) was performed at the heating rate of $10^{\circ} \mathrm{C} / \mathrm{min}$. in nitrogen atmosphere. Wilma et $a l^{2}$., studied kinetics and thermal decomposition of $\mathrm{Cu}(\mathrm{II})$ complex of hydroxyl quinoline-5-sulphonic acid. Thermal data have been analyzed by Freeman-Carroll and Sharp-Wentworth method. Thermal analysis (TGA and DTG) is a typical analytical technique to describe the relationship between physicochemical changes and temperature ${ }^{1-2}$ in order to synthesize complexes having practical applications. There is a need to investigate the effect of heat on complexes in order to establish thermal stability. Iqbal et al., ${ }^{3-7}$ have synthesized and characterized complexes of tolbutamide and glibenclamide by FT-IR, elemental analysis and TGA-DTA technique. Thermal studies of complexes were 
carried out to determine their mode of decomposition, the activation energy $\left(\mathrm{E}_{\mathrm{a}}\right)$, order of reaction $(n)$, entropy change $(\Delta S)$, free energy $(\Delta \mathrm{F})$. The Freeman-Carroll and SharpWentworth methods have been used to calculate thermal activation energy and thermal stability. However, very little work has been carried out on the synthesis and characterization and thermal degradation studies of the metal complex of gliclazide. Hence in this work we have prepared the complex of $\mathrm{Cu}$ (II) with gliclazide drug. The solid complex was characterized using different physicochemical methods, like elemental analysis (C, H, N, S and metal content), IR, NMR and thermal analysis (TGA and DTG).

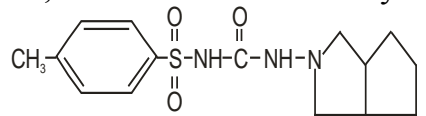

\section{Experimental}

Figure 1. Structure of gliclazide

\section{Ligand-metal ratio}

a) Gliclazide $0.005 \mathrm{M}$ solution was prepared by dissolving $1.617 \mathrm{~g}$ in $100 \mathrm{~mL}$ ethanol, and cupric chloride solution was prepared by dissolving $0.426 \mathrm{~g}$ in $100 \mathrm{~mL}$ of ethanol. $10 \mathrm{~mL}$ gliclazide solution was titrated conductometrically against cupric chloride solution taken in burette. Conductance was recorded after each addition with proper stirring at temperature $30 \pm 1{ }^{\circ} \mathrm{C}$. Results were plotted between corrected conductance and volume of metal salt. From the equivalent point in the graph, ratio between metal and ligand was noted to be 1:2.

b) Formation of 2:1 $\left(\mathrm{L}_{2} \mathrm{M}\right)$ ratio was further confirmed by Job's method ${ }^{8}$ of continuous variation as modified by Turner and Anderson ${ }^{9}$ (Table 1 and Figure $2 \mathrm{a} \& 2 \mathrm{~b}$ ). Spectrophotometric studies were conducted using absorbance as index property, from these values the stability constant $(\log \mathrm{k})$ and free energy change $(\Delta \mathrm{F})$ were also calculated for gliclazide-copper complex.

Table 1. Gliclazide with copper (Job's method)

\begin{tabular}{cccccc}
\hline S.No. & Metal:Ligand & \multicolumn{2}{c}{ Absorbance } & \multicolumn{2}{c}{ Corrected Absorbance } \\
& ratio & $0.002 \mathrm{M}$ & $0.005 \mathrm{M}$ & $0.002 \mathrm{M}$ & $0.005 \mathrm{M}$ \\
\hline 1 & $0: 12$ & 0.006 & 0.01 & 0.00 & 0.00 \\
2 & $1: 11$ & 0.065 & 0.095 & 0.059 & 0.085 \\
3 & $2: 10$ & 0.112 & 0.135 & 0.106 & 0.125 \\
4 & $3: 9$ & 0.167 & 0.19 & 0.161 & 0.175 \\
5 & $4: 8$ & 0.205 & 0.260 & 0.198 & 0.241 \\
6 & $5: 7$ & 0.185 & 0.240 & 0.178 & 0.220 \\
7 & $6: 6$ & 0.145 & 0.198 & 0.138 & 0.185 \\
8 & $7: 5$ & 0.098 & 0.123 & 0.090 & 0.109 \\
9 & $8: 4$ & 0.090 & 0.112 & 0.082 & 0.099 \\
10 & $9: 3$ & 0.052 & 0.075 & 0.044 & 0.065 \\
11 & $10: 2$ & 0.033 & 0.043 & 0.025 & 0.029 \\
12 & $11: 1$ & 0.021 & 0.025 & 0.020 & 0.019 \\
13 & $12: 0$ & 0.009 & 0.014 & 0.00 & 0.00 \\
\hline
\end{tabular}

Synthesis of Complex

The chemicals used in this synthesis were all of Analar Grade Hi-media. A weighed quantity of gliclazide $(2 \mathrm{~mol})$ (supplied by Zim laboratory Nagpur, India) was dissolved separately in minimum quantity of $90 \%$ ethanol. The cupric chloride solution was prepared by dissolving ( 1 mole) separately in the same solvent. Ligand solution was added slowly with stirring into the metallic salt solution at room temperature. The $\mathrm{pH}$ was maintained between 6.0 and 6.5 by adding 
dilute $\mathrm{NaOH}$ solution. On refluxing the mixture for $3 \mathrm{~h}$ and on cooling the complex separated out, filtered off, washed well with ethanol and finally dried in vacuum and weighed.

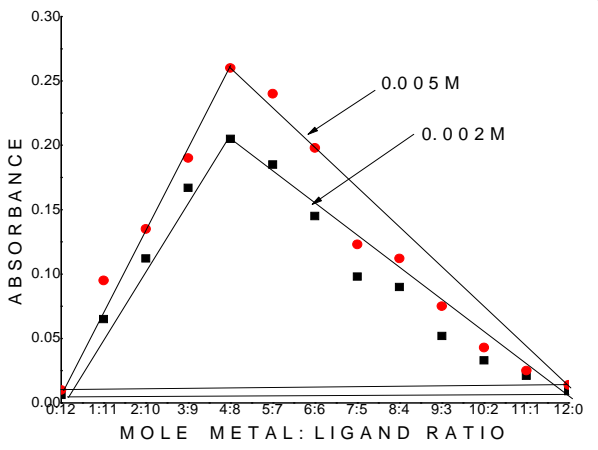

Figure 2a.

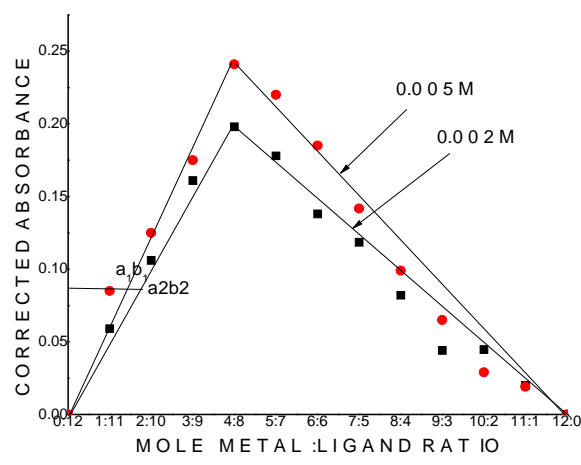

Figure 2b.

Job's Method of continuous variation (Modified by Turner and Anderson)

\section{Instrumentation}

The elemental analysis of the isolated complex was carried out using Coleman Analyzer at the Departmental Microanalytical Laboratory CDRI Lucknow. The IR spectra of the ligand as well as of the complex was recorded on Perkin Elemer Spectrometer and ${ }^{1} \mathrm{H}$ NMR spectra of the ligand and isolated complex was recorded on a Bruker DRX-300 spectrometer and $\mathrm{d}_{6}$-DMSO was used as a solvent. IR and ${ }^{1} \mathrm{H}$ NMR spectra recorded in CDRI, Lucknow and IIT Bombay, India.

From stoichoimetry and analytical data (Table 2), the composition of the complex comes out to be $\left(\mathrm{C}_{15} \mathrm{H}_{21} \mathrm{~N}_{3} \mathrm{O}_{3} \mathrm{~S}\right)_{2} \cdot \mathrm{Cu}$, for, which favors 2:1 $\left(\mathrm{L}_{2} \mathrm{M}\right)$ ratio. The tentative structure (c.f. Figure 7) was assigned to complex on the basis of analytical data and IR, NMR.

\section{Results and Discussion}

Table 2. Analytic and physical data of gliclazide metal complex

\begin{tabular}{|c|c|c|c|c|c|c|c|c|}
\hline S. No. & Complex & Color & $\begin{array}{c}\text { Yield } \\
\%\end{array}$ & $\begin{array}{c}\text { m.p } \\
{ }^{\circ} \mathrm{C}\end{array}$ & $-\Delta \mathrm{F}$ & $\log \mathrm{K}$ & $\begin{array}{c}\text { Molar } \\
\Omega^{-1} \\
\end{array}$ & $\begin{array}{l}\text { lductance } \\
{ }^{-1} \mathrm{~mole}^{-1}\end{array}$ \\
\hline \multirow{4}{*}{1.} & \multirow{4}{*}{$\left(\mathrm{C}_{15} \mathrm{H}_{21} \mathrm{~N}_{3} \mathrm{O}_{3} \mathrm{~S}\right)_{2} \mathrm{Cu}$} & $\begin{array}{c}\text { Maple } \\
\text { green }\end{array}$ & 58.79 & 169 & 16.65 & 12.02 & & .1 \\
\hline & & \multirow{2}{*}{ M. Wt. } & \multicolumn{6}{|c|}{ Analytical data calculated (theoretical), $\%$} \\
\hline & & & $\mathrm{C}$ & & $\mathrm{H}$ & $\mathrm{N}$ & $\mathrm{S}$ & Metal \\
\hline & & 708 & $\begin{array}{r}50.19 \\
(50.50\end{array}$ & & $\begin{array}{l}.00 \\
.01)\end{array}$ & $\begin{array}{c}10.47 \\
(11.47)\end{array}$ & $\begin{array}{c}9.00 \\
(8.47)\end{array}$ & $\begin{array}{c}7.99 \\
(7.75)\end{array}$ \\
\hline
\end{tabular}

\section{Infra-Red Spectra Studies}

The IR spectra of ligand and isolated complex (Figure 3) were scanned within the range $4000-400 \mathrm{~cm}^{-1}$. Assignments (Table 3) of the infrared spectral bands are based on literature. IR spectrum shows important bands due to $v(\mathrm{M}-\mathrm{O})$ 400-600 $\mathrm{cm}^{-1}, v(\mathrm{Ar}-\mathrm{S}) 700-800 \mathrm{~cm}^{-1}$, $v(-\mathrm{S}-\mathrm{N}) 1085 \pm 20 \mathrm{~cm}^{-1}, v\left(\mathrm{SO}_{2}-\mathrm{N}\right) 1120 \pm 20 \mathrm{~cm}^{-1}, v(\mathrm{C}-\mathrm{N}) 1210 \pm 20 \mathrm{~cm}^{-1}, v(\mathrm{~S}=\mathrm{O}) 1340 \pm 20$ $\mathrm{cm}^{-1}, v(\mathrm{C}=\mathrm{O}) 1710 \mathrm{~cm}^{-1}, v(\mathrm{NH}-\mathrm{stretch}) 3274 \pm 20 \mathrm{~cm}^{-1}$. The proposed structure for the isolated complex is also supported by IR absorption, C.N.Rao ${ }^{10}$, Bellamy ${ }^{11}$ and Weissberger ${ }^{12}$

\section{${ }^{1}$ H NMR Studies}

${ }^{1} \mathrm{H}$ NMR spectral data are given in Table 4. It is observed that the singlet due to the imide (NH) proton around $\delta 8.033$ in the spectrum of the ligand but disappeared in the spectra of $\mathrm{NH}$ group in 
the complex molecule due to formation of $\mathrm{M}-\mathrm{O}$ band. This is also confirms the deprotonation of imide $(\mathrm{NH})$ group through enolization (the appearance of $>\mathrm{C}=\mathrm{N}$ stretching band observed in $\mathrm{IR}$ spectra). Other features of NMR spectrum were the aromatic presence of unresolved multiplet suggestive protons ${ }^{13-17}$.

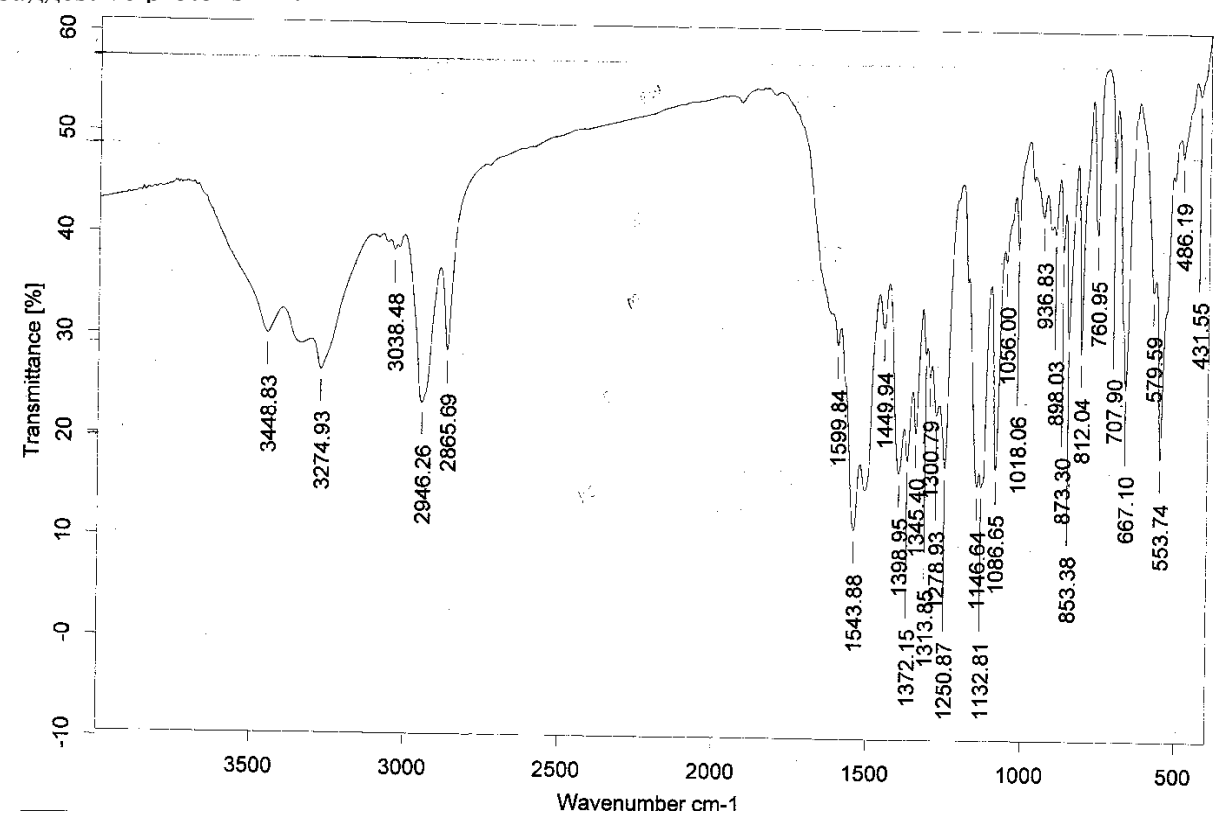

Figure 3. IR spectra of gliclazide-copper complex

\section{Thermal study of gliclazide-copper complex}

In the present investigation, the weight loss of the complex was calculated within corresponding temperature ranges. The obtained data are listed in Table 5. The complex is thermally decomposed in three decomposition steps within the temperature range of $50-600{ }^{\circ} \mathrm{C}$. The TGA/DTA curves for the complexes are shown in Figure 4.

Table 3. Specific IR assignment of gliclazide(ligand) and gliclazide-copper complex

\begin{tabular}{l|l}
\hline Pure drug (Gliclazide) & Gliclazide-Cu complex \\
\hline $632 \mathrm{~cm}^{-1}(\mathrm{~s}), 668 \mathrm{~cm}^{-1}(\mathrm{vs}), 1087 \mathrm{~cm}^{-1}(\mathrm{vs})$, & $547 \mathrm{~cm}^{-1}(\mathrm{~s}), 753(\mathrm{vs}), 1122 \mathrm{~cm}^{-1}(\mathrm{~s}), 1216 \mathrm{~cm}^{-1}(\mathrm{vs})$, \\
$1240 \mathrm{~cm}^{-1}(\mathrm{vs}), 1348 \mathrm{~cm}^{-1}(\mathrm{vs}), 1710 \mathrm{~cm}^{-1}(\mathrm{vs})$, & $1622(\mathrm{vs}) 1337 \mathrm{~cm}^{-1}(\mathrm{~s}), 1524 \mathrm{~cm}^{-1}(\mathrm{~s}) 2947 \mathrm{~cm}^{-1}(\mathrm{~m})$, \\
$2867 \mathrm{~cm}^{-1}, 2950 \mathrm{~cm}^{-1}(\mathrm{vs}), 3274 \mathrm{~cm}^{-1}(\mathrm{vs})$, & $3021 \mathrm{~cm}^{-1}(\mathrm{~s}), 3674 \mathrm{~cm}^{-1}(\mathrm{~m})$ \\
$\mathrm{vs}=$ very strong, $\mathrm{s}=$ strong & vs = very strong, $\mathrm{s}=$ strong, $\mathrm{m}=$ medium \\
\hline
\end{tabular}

Table 4. NMR Assignments of gliclazide-copper complex

\begin{tabular}{ll}
\hline \multicolumn{1}{c}{$\left(\mathrm{C}_{15} \mathrm{H}_{21} \mathrm{~N}_{3} \mathrm{O}_{3} \mathrm{~S}\right)_{2}$ pure drug Gliclazide } & \multicolumn{1}{c}{$\left(\mathrm{C}_{15} \mathrm{H}_{20} \mathrm{~N}_{3} \mathrm{O}_{3} \mathrm{~S}\right)_{2} \mathrm{Cu}$} \\
\hline$\delta 8.041\left(\mathrm{~s}, 1 \mathrm{H}, \mathrm{NHCO}, \mathrm{J}=0.334 \mathrm{H}_{\mathrm{z}}\right), \delta 7.817$ & $\delta 7.691\left(\mathrm{~d}, 1 \mathrm{H}, \mathrm{NH}-\mathrm{CO}, \mathrm{J}=1 \mathrm{H}_{\mathrm{z}}\right), \delta 7.346(\mathrm{~d}$, \\
$\left(\mathrm{d}\right.$, benzene $\left.\mathrm{J}=1 \mathrm{H}_{\mathrm{z}}\right), \delta 7.395(\mathrm{~d}$, benzene, & benzene, $\left.\mathrm{J}=0.69 \mathrm{H}_{\mathrm{z}}\right), \delta 6.328\left(\mathrm{~s}, \mathrm{SO}_{2}-\mathrm{NH}\right.$, \\
$\left.\mathrm{J}=1 \mathrm{H}_{\mathrm{z}}\right), \delta 6.28\left(\mathrm{~s}, \mathrm{SO}_{2} \mathrm{NH}\right), \delta 3.320(\mathrm{NH}-$ & $\left.\mathrm{J}=1 \mathrm{H}_{\mathrm{z}}\right), \delta 3.668\left(\mathrm{~s}, \mathrm{NH}-\mathrm{CO}-\mathrm{Cu}, \mathrm{J}=0.83 \mathrm{H}_{\mathrm{z}}\right)$, \\
$\left.\mathrm{CO}, \mathrm{J}=0.929 \mathrm{H}_{\mathrm{z}}\right), \delta 2.901\left(\mathrm{~s}, \mathrm{CH}_{3}\right.$ group & $\delta 2.724\left(\mathrm{~s}, \mathrm{CH}_{3}\right.$-group attached to benzene, \\
attached to benzene, $\left.\mathrm{J}=2.160 \mathrm{H}_{\mathrm{z}}\right), \delta 1.388$ & $\left.\mathrm{~J}=1.26 \mathrm{H}_{\mathrm{z}}\right), \delta 1.608\left(\mathrm{q}, \mathrm{CH}_{3}\right.$ group, $\left.\mathrm{J}=5.50 \mathrm{H}_{\mathrm{z}}\right)$, \\
$\left(\mathrm{s}, \mathrm{CH}_{3}\right.$ group, $\mathrm{J}=2.955 \mathrm{H}_{\mathrm{z}}$ & $\delta 1.009\left(\mathrm{CH}_{3}\right.$ group, $\mathrm{J}=2.03 \mathrm{H}_{\mathrm{Z}}$ \\
\hline
\end{tabular}
$\mathrm{s}=$ singlet, $\mathrm{d}=$ doublet, $\mathrm{t}=$ triplet, $\mathrm{q}=$ quartrate, $\mathrm{m}=$ multiple 


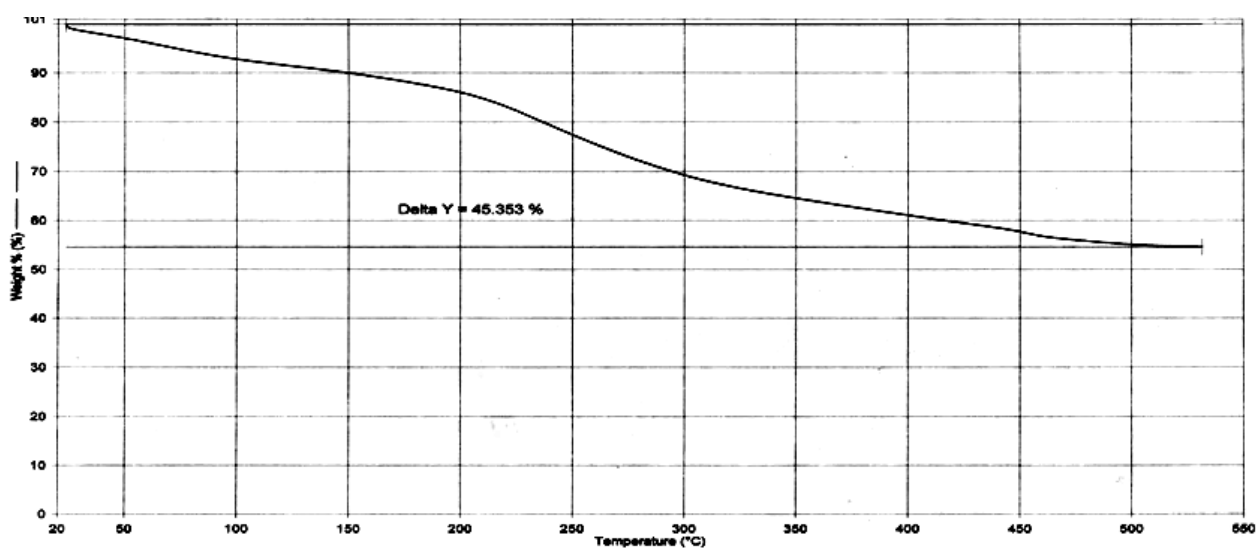

Figure 4. TGA curve of gliclazide -copper complex

The thermoanalytical data are presented in Table 6. In studying the decomposition kinetics methods mentioned in the literature was used in each case the least square plots were drawn. The first few points that did not fall on straight line were discarded. These types of deviations of points are reported in literature by several research workers ${ }^{20-29}$.

\section{Theoretical Consideration}

To provide further evidence regarding the degradation system of analyzed compounds, we derived the TG curves by applying an analytical method proposed by Freeman-Carroll ${ }^{27}$ and Sharp-Wentworth. ${ }^{28}$

\section{Freeman-Carroll Method}

The straight line equation derived by Freeman and Carroll is:

$$
\frac{\Delta \log \frac{d w}{d t}}{\Delta \log W r}=n-\frac{E a}{2.303 R} \frac{\Delta \frac{1}{T}}{\Delta \log W r}
$$

Where, $\frac{d w}{d t}=$ rate of change of weight with time, $W r=W c-W, W_{c}=W t$. loss at completion of reaction, $W=$ Total wt. loss up to time ' $\mathrm{t}$ ', $\mathrm{E}_{\mathrm{a}}=$ Energy of activation, $\mathrm{n}=$ Order of reaction.

The plot between the term $\frac{\Delta \log \frac{d w}{d T}}{\Delta \log W r}$ vs $\frac{\Delta \frac{1}{T}}{\Delta \log W r}$ gives a straight line (Figure 5) from which slope can be calculated. The energy of activation $\left(E_{a}\right)$ and intercept on $Y$-axis as order of reaction $(\mathrm{n})$, the change of entropy $(\Delta \mathrm{S})$ are also can be calculated.

\section{Sharp-Wentworth Method}

Using the Sharp and Wentworth equation:

$$
\frac{\Delta \log \frac{d c}{d t}}{(1-c)}=\log \frac{A}{\beta}-\frac{E a}{2.303 R} \frac{1}{T}
$$

Where, $\frac{d c}{d t}=$ Rate of change of fraction of weight with change in temperature. $\beta=$ Linear heating rate $\frac{d T}{d t}$, a graph was plotted between $\frac{\Delta \log \frac{d c}{d t}}{(1-c)} v s \frac{1}{T}$. We obtained a straight line

(Figure 6) and the energy of activation $\left(\mathrm{E}_{\mathrm{a}}\right)$ was calculated from its slope. 


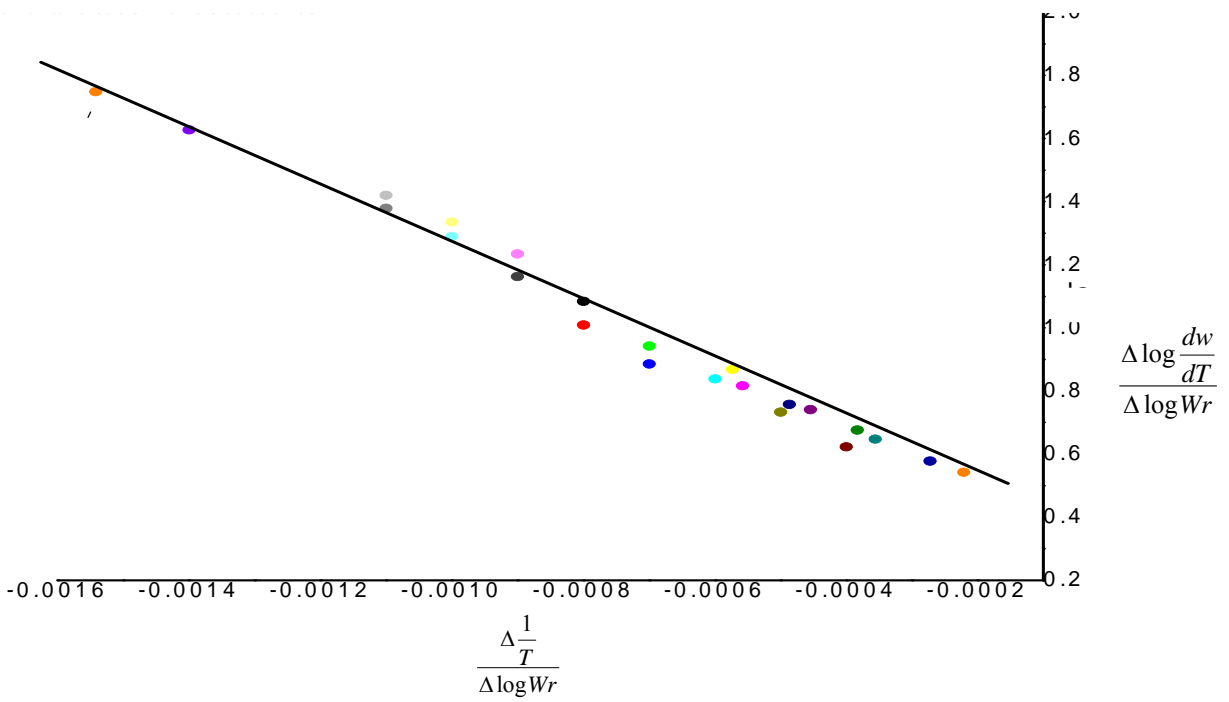

Figure 5. Determination of order of reaction and activation energy by FC method

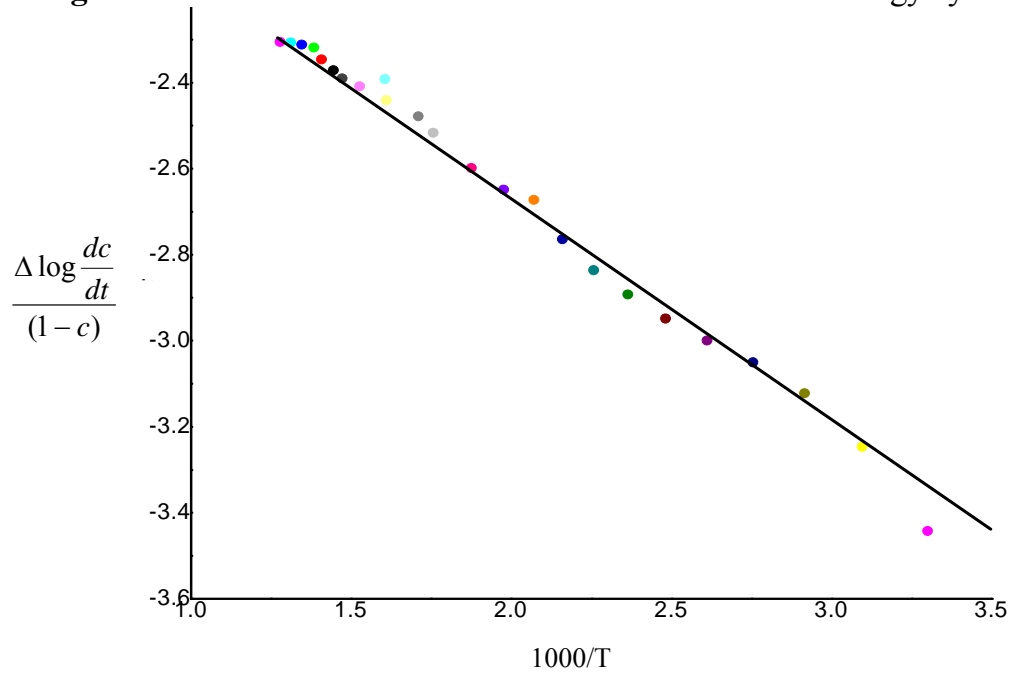

Figure 6. Determination of activation energy by SW method

The thermodynamic activation parameters of decomposition process of dehydrate complexes namely activation energy $\left(E_{a}\right)$, Entropy $(\Delta S)$ and Gibb's free energy change of decomposition $\left(\Delta \mathrm{G}^{\circ}\right)$ were evaluated graphically by employing Freeman-Carroll and SharpWentworth relation. The data are summarized in Table 7 . The activation energies of decomposition are found to be in the range 54 to $56 \mathrm{~kJ} \mathrm{~mole}^{-1}$. The high value of activation energies reflects the thermal stability of complex. The entropy of activation is found to have negative values in complex which indicate that decomposition reactions process with lower rate than the normal ones, beside these parameters, in TGA curve (Figure 5), the initial loss with in temperature $20-450{ }^{\circ} \mathrm{C}$ followed by loss of ligand molecule in the next step within the temperature range $\geq 450{ }^{\circ} \mathrm{C}$ with the formation of metal oxide as a residue of decomposition. 
Table 5. Thermogravimetric data of gliclazide metal complex by Sharp-Wenrworth method

\begin{tabular}{|c|c|c|c|c|c|c|c|c|c|c|}
\hline $\begin{array}{c}\text { Temp. } \\
{ }^{\circ} \mathrm{C}\end{array}$ & $\begin{array}{c}\text { Temp } \\
{ }^{\circ} \mathrm{K}\end{array}$ & $\frac{1000}{T}$ & $\begin{array}{c}\% \\
\text { Mass } \\
\text { Loss } \\
\end{array}$ & $\begin{array}{c}\text { Change } \\
\text { in } W t . \text { ' }^{\prime} \text { ' } \\
\mathrm{g}\end{array}$ & $1-c$ & $\frac{d c}{d t}$ & $\log \frac{d c}{d t}$ & $\log (1-c)$ & $\frac{\log \frac{d c}{d t}}{(1-c)}$ & $\begin{array}{c}\text { Weight } \\
\%\end{array}$ \\
\hline 30 & 303 & 3.30033 & 1.381 & 0.00019 & 0.99981 & 0.00036 & -3.44273 & -0.00008 & -3.44338 & 98.619 \\
\hline 50 & 323 & 3.09598 & 2.928 & 0.00040 & 0.99960 & 0.00057 & -3.24651 & -0.00017 & -3.24780 & 97.072 \\
\hline 70 & 343 & 2.91545 & 4.752 & 0.00065 & 0.99935 & 0.00075 & -3.12209 & -0.00028 & -3.12411 & 95.248 \\
\hline 90 & 363 & 2.75482 & 6.499 & 0.00088 & 0.99912 & 0.00089 & -3.05055 & -0.00038 & -3.05325 & 93.501 \\
\hline 110 & 383 & 2.61097 & 7.842 & 0.00107 & 0.99893 & 0.00100 & -2.99942 & -0.00046 & -3.00262 & 92.158 \\
\hline 130 & 403 & 2.48139 & 8.928 & 0.00121 & 0.99879 & 0.00113 & -2.94832 & -0.00053 & -2.95191 & 91.072 \\
\hline 150 & 423 & 2.36407 & 10.064 & 0.00137 & 0.99863 & 0.00128 & -2.89309 & -0.00060 & -2.89705 & 89.936 \\
\hline 170 & 443 & 2.25734 & 11.414 & 0.00155 & 0.99845 & 0.00146 & -2.83590 & -0.00067 & -2.84032 & 88.586 \\
\hline 190 & 463 & 2.15983 & 13.007 & 0.00177 & 0.99823 & 0.00172 & -2.76460 & -0.00077 & -2.76951 & 86.993 \\
\hline 210 & 483 & 2.07039 & 15.239 & 0.00207 & 0.99793 & 0.00213 & -2.67264 & -0.00090 & -2.67819 & 84.761 \\
\hline 230 & 503 & 1.98807 & 18.666 & 0.00254 & 0.99746 & 0.00256 & -2.59115 & -0.00110 & -2.59774 & 81.334 \\
\hline 250 & 523 & 1.91205 & 22.575 & 0.00307 & 0.99693 & 0.00295 & -2.52985 & -0.00134 & -2.53764 & 77.425 \\
\hline 270 & 543 & 1.84162 & 26.213 & 0.00357 & 0.99643 & 0.00328 & -2.48401 & -0.00155 & -2.49290 & 73.787 \\
\hline 290 & 563 & 1.77620 & 29.356 & 0.00399 & 0.99601 & 0.00355 & -2.45015 & -0.00174 & -2.45997 & 70.644 \\
\hline 310 & 583 & 1.71527 & 31.94 & 0.00435 & 0.99565 & 0.00374 & -2.42724 & -0.00189 & -2.43783 & 68.060 \\
\hline 330 & 603 & 1.65837 & 33.869 & 0.00461 & 0.99539 & 0.00390 & -2.40885 & -0.00201 & -2.42001 & 66.131 \\
\hline 350 & 623 & 1.60514 & 35.443 & 0.00482 & 0.99518 & 0.00405 & -2.39225 & -0.00210 & -2.40384 & 64.557 \\
\hline 370 & 643 & 1.55521 & 36.875 & 0.00502 & 0.99498 & 0.00420 & -2.37703 & -0.00218 & -2.38901 & 63.125 \\
\hline 390 & 663 & 1.50830 & 38.224 & 0.00520 & 0.99480 & 0.00435 & -2.36200 & -0.00226 & -2.37435 & 61.776 \\
\hline 410 & 683 & 1.46413 & 39.58 & 0.00539 & 0.99461 & 0.00448 & -2.34843 & -0.00235 & -2.36114 & 60.420 \\
\hline 430 & 703 & 1.42248 & 40.865 & 0.00556 & 0.99444 & 0.00464 & -2.33339 & -0.00242 & -2.34643 & 59.135 \\
\hline 450 & 723 & 1.38313 & 42.283 & 0.00575 & 0.99425 & 0.00480 & -2.31835 & -0.00251 & -2.33177 & 57.717 \\
\hline 470 & 743 & 1.34590 & 43.768 & 0.00596 & 0.99404 & 0.00488 & -2.31180 & -0.00259 & -2.32565 & 56.232 \\
\hline 490 & 763 & 1.31062 & 44.602 & 0.00607 & 0.99393 & 0.00493 & -2.30734 & -0.00264 & -2.32143 & 55.398 \\
\hline 510 & 783 & 1.27714 & 45.139 & 0.00614 & 0.99386 & 0.00495 & -2.30544 & -0.00268 & -2.31969 & 54.861 \\
\hline
\end{tabular}


Table 6. Thermogravimetric data of gliclazide metal complex by Freeman-Corroll method

\begin{tabular}{|c|c|c|c|c|c|c|c|c|c|c|c|c|c|c|c|c|c|}
\hline 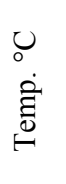 & $\begin{array}{l}n \\
0 \\
0 \\
0 \\
0 \\
\tilde{J} \\
\sum^{0} \\
0\end{array}$ & 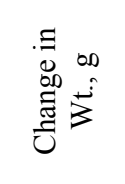 & $\stackrel{n}{\Xi}$ & $\frac{d w}{d t}$ & $\log \frac{d w}{d t}$ & $\begin{array}{c}W r= \\
W c-W\end{array}$ & $\begin{array}{l}\log \\
\mathrm{Wr}\end{array}$ & $\stackrel{v}{H}$ & $\frac{1}{T}\left(K^{-1}\right)$ & $\frac{\log \frac{d w}{d t}}{\log w r}$ & $\frac{\log \frac{d w}{d t}}{\log w r}$ & $\alpha=\frac{w t}{w c}$ & 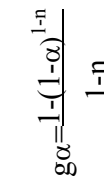 & $\begin{array}{c}\hat{0} \\
x \\
n^{x}\end{array}$ & 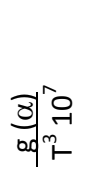 & $\frac{1}{T} \times 10^{-3}$ & $\log \left(\frac{g(\alpha)}{T^{3}}\right)$ \\
\hline 30 & 1.381 & 00019 & 90 & 000389 & .410 & 00599 & -2.2226 & 303 & 0 & 1.534 & .0015 & 0304 & .0309 & 2.7818 & $\overline{\mathrm{an}}$ & .3003 & -108.8234 \\
\hline 50 & 2.928 & 0.00040 & 150 & 0.000627 & -3.203 & 0.00578 & -2.2381 & 323 & 0.00310 & 1.431 & -0.0014 & 0.0645 & 0.0666 & 3.3698 & 0013 & 3.0960 & -70.2398 \\
\hline 70 & 4.752 & 0.00065 & 210 & 0.000852 & -3.070 & 0.00553 & -2.2572 & 343 & 0.00292 & 1.360 & -0.0013 & 0.1047 & 0.1104 & 4.0354 & 0.0029 & 2.9155 & -48.0044 \\
\hline 90 & 6.499 & 0.00088 & 270 & 0.001023 & -2.990 & 0.00529 & -2.2763 & 363 & 0.00275 & 1.314 & -0.0012 & 1431 & 0.1542 & 4.7832 & .0046 & & -34.6226 \\
\hline 110 & 7.842 & 0.00107 & 330 & 0.001161 & -2.935 & 0.00511 & -2.2915 & 383 & 0.00261 & 1.281 & -0.0011 & 0.1727 & 0.1892 & 5.6182 & 0.0058 & 2.6110 & -26.4437 \\
\hline 130 & 8.928 & 0.00121 & 390 & 0.001309 & -2.883 & 0.00496 & -2.3043 & 403 & 0.00248 & 1.251 & -0.0011 & 0.1966 & 0.2185 & 6.5451 & 0.0066 & 2.4814 & -20.8855 \\
\hline 150 & 10.064 & .00137 & 450 & 0.001485 & -2.828 & 0.00481 & -2.3180 & 423 & 0.00236 & 1.220 & -0.0010 & 2216 & 0.2500 & 7.5687 & .0073 & 2.3641 & -16.6010 \\
\hline 170 & 11.414 & 0.00155 & 510 & 0.001692 & -2.772 & 0.00462 & -2.3349 & 443 & 0.00226 & 1.187 & -0.0010 & 0.2514 & 0.2887 & 8.6938 & 0.0083 & 2.2573 & -13.1039 \\
\hline 190 & 13.007 & 0.00177 & 570 & 0.001985 & -2.702 & 0.00441 & -2.3558 & 463 & 0.00216 & 1.147 & -0.0009 & 0.2865 & 0.3364 & 9.9253 & 0.0097 & 2.1598 & -10.2373 \\
\hline 210 & 15.239 & 0.00207 & 630 & 0.002436 & -2.613 & 0.00410 & -2.3868 & 483 & 0.00207 & 1.095 & -0.0009 & 0.3356 & 0.4072 & 11.2679 & .0121 & 2.0704 & -7.6705 \\
\hline 230 & 18.666 & 0.00254 & 690 & 0.002945 & -2.531 & 0.00364 & -2.4391 & 503 & 0.00199 & 1.038 & -0.0008 & 0.4111 & 0.5267 & 12.7264 & 170 & 1.9881 & -5.2213 \\
\hline 250 & 22.575 & 0.00307 & 750 & 0.003413 & -2.467 & 0.00311 & -2.5078 & 523 & 0.00191 & 0.984 & -0.0008 & 0.4972 & 0.6828 & 14.3056 & 37 & 1.9120 & -3.2795 \\
\hline 270 & 26.213 & 0.00357 & 810 & 0.003816 & -2.418 & 0.00261 & -2.5831 & 543 & 0.00184 & 0.936 & -0.0007 & 0.5773 & 0.8538 & 16.0103 & 308 & 1.8416 & -1.9191 \\
\hline 290 & 29.356 & 0.00399 & 870 & 0.004146 & -2.382 & 0.00218 & -2.6608 & 563 & 0.00178 & 0.895 & -0.0007 & 0.6465 & 1.0292 & 17.8454 & & 1.7762 & -0.9913 \\
\hline 310 & 31.94 & 0.00435 & 930 & 0.004391 & -2.357 & 0.00183 & -2.7371 & 583 & 0.00172 & 0.861 & -0.0006 & 0.7034 & 1.2009 & 19.8155 & 26 & 1. & -0.3698 \\
\hline 330 & 33.869 & 0.00461 & 990 & 0.004592 & -2.338 & 0.00157 & -2.8042 & 603 & 0.00166 & 0.834 & -0.0006 & 0.7459 & 1.3515 & 21.9256 & .0460 & 1.6584 & 0.0161 \\
\hline 350 & 35.443 & 0.00482 & 1050 & 0.004776 & -2.321 & 0.00136 & -2.8679 & 623 & 0.00161 & 0.809 & -0.0006 & 0.7806 & 1.4941 & 24.1804 & 0.0482 & 1.6051 & 0.2762 \\
\hline 370 & 36.875 & 0.00502 & 1110 & 0.004950 & -2.305 & 0.00116 & -2.9353 & 643 & & 0.785 & -0.0005 & 0.8121 & 1.6444 & 26.5848 & & 1.5552 & 0.4726 \\
\hline 390 & 38.224 & 0.00520 & 1170 & 0.005125 & -2.290 & 0.00098 & -3.0101 & 663 & 0.00151 & 0.761 & -0.0005 & 0.8418 & 1.8106 & 29.1434 & 0.0523 & 1.5083 & 0.6281 \\
\hline 410 & 39.58 & 0.00539 & 1230 & 0.005291 & -2.276 & 0.00079 & -3.1010 & 683 & 0.00146 & 0.734 & -0.0005 & 0.8717 & 2.0119 & 31.8612 & 0.0550 & 1.4641 & 0.7657 \\
\hline 430 & 40.865 & 0.00556 & 1290 & 0.005475 & -2.262 & 0.00062 & -3.2092 & 703 & 0.00142 & 0.705 & -0.0004 & 0.9000 & 2.2505 & 34.7429 & 0.0583 & 1.4225 & 0.8823 \\
\hline 450 & 42.283 & 0.00575 & 1350 & 0.005667 & -2.247 & 0.00042 & -3.3718 & 723 & 0.00138 & 0.666 & -0.0004 & 0.9312 & 2.6067 & 37.7933 & 0.0642 & 1.3831 & 1.0191 \\
\hline 470 & 43.768 & 0.00596 & 1410 & 0.005771 & -2.239 & 0.00022 & -3.6522 & 743 & 0.00135 & 0.613 & -0.0004 & 0.9639 & 3.2148 & 41.0172 & 0.0756 & 1.3459 & 1.1975 \\
\hline 490 & 44.602 & 0.00607 & 1470 & 0.005838 & -2.234 & 0.00011 & -3.9616 & 763 & 0.00131 & 0.564 & -0.0003 & 0.9823 & 3.8765 & 44.4195 & 0.0857 & 1.3106 & 1.3073 \\
\hline 510 & 45.139 & 0.00614 & 1530 & 0.005871 & -2.231 & 0.00004 & -4.4414 & 783 & 0.00128 & 0.502 & -0.0003 & 0.9941 & 4.8845 & 48.0049 & 0.1012 & 1.2771 & 1.4296 \\
\hline
\end{tabular}


Table 7. Thermal analyses result of gliclazide complex.

\begin{tabular}{cccccccc}
\hline Complexes & $\begin{array}{c}\text { Decomposition } \\
\text { Temp. }\end{array}$ & \multicolumn{2}{c}{$\mathrm{C}$} & lost. & \multicolumn{2}{c}{$\mathrm{E}_{\mathrm{a}}, \mathrm{J} / \mathrm{mole}$} & \multicolumn{2}{c}{$\Delta \mathrm{S}$} & \multicolumn{2}{c}{$\Delta \mathrm{G}$} & \\
& T.C. & S.W. & J/k/mole & $\mathrm{kJ} / \mathrm{mole}$ & $\mathrm{n}$ \\
\hline$\left(\mathrm{C}_{15} \mathrm{H}_{20} \mathrm{~N}_{3} \mathrm{O}_{3} \mathrm{~S}\right)_{2} \mathrm{Cu}$ & $40-510$ & 45 & 54.89 & 56.48 & -77.31 & 23.08 & 0.55 \\
\hline
\end{tabular}

For supporting the proposed structure of copper-gliclazide complex, initially Job's method of continuous variation as modified by Turner and Anderson was conducted which indicate 2:1 ligand:metal ratio of the complex, moreover stability constant and free energy change was also calculated. Analytical data (c.f. Table 2) agrees to the molecular formula $\left(\mathrm{C}_{15} \mathrm{H}_{20} \mathrm{~N}_{3} \mathrm{O}_{3} \mathrm{~S}\right)_{2} \mathrm{Cu}$. Thermal analysis of complex has been studied by giving their relative thermal stability. Applying the Sharp-Wentworth and Freeman-Corroll methods energy of activation $\left(\mathrm{E}_{\mathrm{a}}\right)$, kinetic parameter viz. $\Delta \mathrm{S}, \Delta \mathrm{G}$ and order of reaction (n) were determine by applying Freeman-Corroll method.

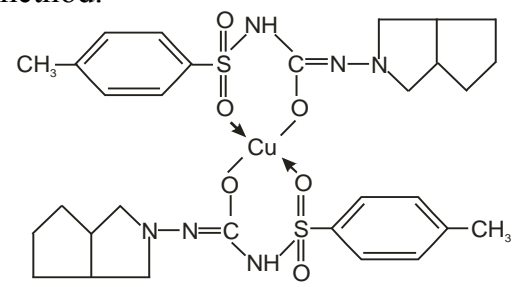

Figure 7. Proposed structure for gliclazide-copper complex

\section{Acknowledgement}

The author is thankful to the principal of Saifia Science College, Bhopal and Principal of Crescent College of Technology, Bhopal, for providing all necessary facilities and I.I.T., Bombay for providing IR and NMR spectra, C.D.R.I, Lucknow for providing analytical data.

\section{References}

1. Bal Krishan, Neeti Rathor and Iqbal S A, International Journal of Theoretical and Applied Sciences, 2013, 5(1), 138-144.

2. Wilma Cyril, Suresh Kumar and Shobhna D, Orient J Chem., 2011, 27(4), 1653-1658.

3. Bal Krishan, Om Prakash and E H EL-Mossalamy, Orient J Chem., 2013, 29(1), 381-388.

4. Neeti Rathore and Bal Krishan, Bioscience Biotechnology Research Asia, 2013, 10(1), 479-487.

5. Iqbal S A, Sibi Jose and Ishaq Zaafarany, Orient J Chem., 2012, 28(1), 613-618.

6. Bal Krishan and Ishaq Zaafarany, Orient J Chem., 2013, 29(4), 1571-1577.

7. Md Tawkir, Iqbal S A, Bal Krishan and Ishaq Zaafarany, Orient J Chem., 2011, 27(2), 603-609.

8. Job P Annales de Chimie., 1928, 10, 113.

9. Turner S E and Anderson R C, J Am Chem Soc., 1949, 71, 912.

10. Rao C N R, Chemical Applications of Infrared Spectroscopy, Academic Press, Newyork, London, 1963.

11. Bellamy L J, The Infrared spectra of complex molecules. Matheun and Co.Ltd. 1954,

12 Weissberger A, Techniques of Organic Chemistry:Chemical Application of Spectroscopy, Interscience Publishers, New York, 1956.

13. Slichter C P, Principles of magnetic resonance, 1963.

14. Akit J W, NMR and Chemistry- An introduction to nuclear magnetic resonance spectroscopy, Champan and Hall: $2^{\text {nd }}$ Review; 1973, 400. 
15 Siewers R E, Nuclear magnetic resonance shift reagents academic, New York, 1973.

16. Neeti Rathore and Bal Krishan, Orient J Chem., 2013, 29(3), 1001-1008.

17. Farhana, Afridi, Iqbal S A and Javed Hasan, Orient J Chem., 2010, 22(1), 195-197.

18. Madhusudhayan P M, Krishan K and Ninan K N, Thermochim Acta., 1986, 97, 189.

19. Pandey R N, Nag A K, Prasashti Pandey and Sanjay K Sing, Orient J Chem., 2010, 26(1), 109-112.

20. Rai B K and Sateydev Singh, Orient J Chem., 2010, 26(3), 989-994.

21. Birendra Kumar, Kiran Kumar Prasad and Sanjay Kumar, Orient J Chem., 2010, 26(4), 1413-1418.

22. Subi A AL-Jibori, Ibrahim F Waheed and Alif Al-Samoraie, Orient J Chem., 2012, 28(1), 257-262.

23. Rehman F and Samya Majraj, Orient J Chem., 2012, 28(1), 581-585.

24. Alhadi A A, Shaker S A, Yehe W A, Ali H M and Mahmood A A, Orient J Chem., 27(4), 2011, 1437-1442.

25. Freeman E S and Correl B, J Phys Chem., 1958, 62(4), 394-397.

26. Sharp J P and Wentworth S A, Anal Chem., 1969, 41(14), 2060-2062.

27. Singru R N, Arch.Appl.Sci. Res., 2011, 3(5), 309-325.

28. Rahangdale S S and Gurnule W B, Chem Sci Trans., 2013, 2(1), 287-293; DOI:10.7598/cst2013.312

29. Bal Krishan and Iqbal S A, J Chem., 2014, p10; DOI: 10.1155/2014/378567 